\title{
Normalizing Relational Database Schemas Using Mathematica
}

\author{
Ali Yazici $^{1}$ and Ziya Karakaya ${ }^{2}$ \\ ${ }^{1}$ Computer Engineering Department, TOBB University of Economics \& Technology, \\ Ankara - Turkey \\ aliyazici@etu.edu.tr \\ ${ }^{2}$ Computer Engineering Department, Atilim University, Ankara - Turkey \\ ziya@atilim.edu.tr
}

\begin{abstract}
In this paper, basic relational database (DB) normalization algorithms are implemented efficiently as Mathematica modules. It was observed that, Mathematica provided a straightforward platform as opposed to previous ones, mainly Prolog based tools which required complex data structures such as linked list representations with pointers. A Java user interface called JMath-Norm was designed to execute the Mathematica modules in a systematic way. For this purpose, Mathematica's Java link facility (JLink) is utilized to drive the Mathematica kernel. JMath-Norm provides an effective interactive tool in an educational setting for teaching DB normalization theory.
\end{abstract}

\section{Introduction}

Conceptual modeling is one of the most important steps in the design of DB systems. It is in a way the transformation of the real life problem or business rules into a set of entities with certain attributes and relationships among them. The ER, EER or UML data modeling tools are utilized for the purpose of representing this transformation graphically. The entity set in the conceptual model is eventually mapped into a set of relation schemas $\left\{R_{1}, R_{2}, R_{3}, \ldots, R_{n}\right\}$ where each $R_{i}$ represents one of the relations (tables) in the DB schema. This mapping is accomplished by using a set of informal rules [1].

The next step in the design process is normalization in which each $R_{i}$ along with its attribute set and a set of functional dependencies defined are considered and the relation schema is decomposed into sub relations to satisfy the so called normalization criteria. In the literature $1 \mathrm{NF}, 2 \mathrm{NF}, 3 \mathrm{NF}, \mathrm{BCNF}, 4 \mathrm{NF}$ and $5 \mathrm{NF}$ are commonly cited even though some other special normal forms do exist.

Although automatic normalization has been a point of attraction for DB researchers from the very beginning of the relational DB theory, commercial DB tools do not provide a complete solution for automatic normalization.

Computational problems and some algorithmic problems in the design of normalized relation schemas has been considered in [2]. A Prolog implementation of the normalization process can be found in [3. Another Prolog based normalization tool is described in 4. Some new and fast algorithms for DB normalization is 
provided in [5, 6] where a new method for computing minimal covers is presented using a modified definition of closure for normalizing relations. Normalization and some associated problems have been investigated from a semantical point of view in [7]. A polynomial time algorithm for finding all possible candidate keys in a relation schema is given in 8 .

Decomposing relations into sub relations during the normalization process normally introduces some overhead in the DB querying. Cost effectiveness of normalization process has been undertaken by some researches [9, 10].

A practical solution to $1 \mathrm{NF}$ problem was given in [1] where the repeating groups are handled by creating an extra superfluous repeater table. A discussion on relations which are in $3 \mathrm{NF}$ but not in BCNF is provided in 12 .

In this paper, the symbolic computational power of Mathematica and the Java are combined to design a normalization tool for DB relation schemas. Basic normalization algorithms are implemented efficiently in Mathematica and Java is utilized to design a windows based application. This tool aims to provide an educational setting for the undergraduate students studying DB systems. All standard normalization algorithms are implemented and can be tested individually or in an integrated fashion to provide $3 \mathrm{NF}$ relations. The design approach in this study is similar to the one given in 13 although the underlying environment and the purpose of the study are totally different.

The basic data structure used in all implementations given here are just lists together with the standard Mathematica set operations as opposed to the linked lists and pointers and complicated programming structures used in the previous implementations (see for example [3, 4, 13]).

The tool designed in this work utilizes JLink [14, Java link mechanism of the Mathematica.

Section 2 briefly discusses the normalization theory. In Section 3 Mathematica implementation of basic normalization algorithms is given. Java interface via JLink is given in Section 4. Final remarks and conclusions are provided in the final section.

\section{Theoretical Background}

As mentioned in the preceding section, the relational DB schema as an outcome of the conceptual modeling should be normalized to sustain the integrity of the DB. The normalization process relies on the concept of functional dependency (FD) between set of attributes of the DB schema. Formally, a FD between two sets of attributes $\mathrm{X}$ and $\mathrm{Y}$, denoted by, $X \rightarrow Y$ specifies that for any two tuples $t_{1}$ and $t_{2}$ in a relation state $\mathrm{r}$ of $R=\left\{A_{1}, A_{2}, \cdots, A_{n}\right\}$ with $\mathrm{n}$ attributes that have $t_{1}[X]=t_{2}[X]$, they must also have $t_{1}[Y]=t_{2}[Y]$. One says that $\mathrm{Y}$ is functionally dependent on $\mathrm{X}$.

For example, for a DB schema named STUDENT $=\{$ studentNo, studentName, instructorId, officeNo\}, with studentNo as the primary key, one can write the following FD's: 
FD1 : studentNo $\rightarrow\{$ studentName, instructorId, officeNo $\}$

FD2 : instructorId $\rightarrow$ officeNo

Other set of FD's can be obtained using the concept of attribute set closure. For each set of attributes $\mathrm{X}$, we determine $X^{+}$, the set of attributes that can be determined by $\mathrm{X}$ based on the set of FD's F of the schema. An algorithm to do so is given below 1 :

\section{Algorithm ClosureX: Determining $X^{+}$under FD}

Given a DB schema R, a set of attributes $\mathrm{X}$ and FD set F calculate $X^{+}$

1. $X^{+}:=X$

2. repeat

$$
\begin{aligned}
& \text { temp } X^{+}:=X^{+} \\
& \text {for each FD } Y \rightarrow Z \text { in } F \text { do } \\
& \quad \text { if } X^{+} \supseteq Y \text { then } X^{+}:=X^{+} \cup Z \\
& \text { until }\left(X^{+}=\text {temp } X^{+}\right)
\end{aligned}
$$

Given a set of FD's F, an attribute B is said to be extraneous 5 ] in $X \rightarrow A$ with respect to $\mathrm{F}$ if $X=Z B, X \neq Z$, and $A \in Z^{+}$. A set of FD's $\mathrm{H}$ is called a minimal cover [1,5] for a set $\mathrm{F}$ if each dependency in $\mathrm{H}$ as exactly one attribute on the right-hand side, if no attribute on the left-hand side is extraneous, and if no dependency in $\mathrm{H}$ can be derived from the other dependencies in $\mathrm{H}$. All normalization algorithms uses the minimal cover of a given set of FD's.

In this study, the closure and minimal cover algorithms are implemented in Mathematica. An algorithm to calculate the minimal cover is given in [1]. Actually, the calculation of a minimal cover consists of "Elimination of Extraneous Attributes" followed by the "Elimination of Redundant Dependencies". Normalization is a step by step procedure in which the original DB schema is decomposed into a set of relations to satisfy the so called normalization conditions. $1 \mathrm{NF}$ relations are those without any multi-valued attributes. In a $2 \mathrm{NF}$ relation there are no partial dependencies. 3NF relations do not contain any transitive dependencies among its attributes. This paper utilizes Bernstein's algorithm below [1,15] to provide $3 \mathrm{NF}$ schemas for a given set of attributes and a set of FD's F. $2 \mathrm{NF}$ condition is automatically satisfied and dependencies are preserved. However, lossless join property [1] is not guaranteed.

Algorithm ThirdNormalForm: Bernstein's Synthesis Algorithm 15] Given a DB schema $\mathrm{R}$ and FD set F, find $3 \mathrm{NF}$ decompositions D

1. Find a Minimal Cover $\mathrm{G}$ for $\mathrm{F}$

2. For each a left-hand side $\mathrm{X}$ of a $\mathrm{FD}$ that appears in $\mathrm{G}$, create a relation schema in D with attributes $\left\{X \cup\left\{A_{1}\right\} \cup\left\{A_{2}\right\} \cup \ldots \cup\left\{A_{k}\right\}\right\}$, where $X \rightarrow A_{1}$, $X \rightarrow A_{2}, \ldots, X \rightarrow A_{k}$ are the only dependencies in $\mathrm{G}$ with $\mathrm{X}$ as the left-hand side ( $\mathrm{X}$ is the key)

3. Place any remaining attributes (that have not been placed in any relation) in a single relation schema to ensure the attribute preservation 


\section{Mathematica Implementation and Results}

\subsection{NF Normalization with Mathematica}

In this section implementation of the Bernstein's 3NF algorithm in Mathematica is discussed. The use case diagram in Fig.1 shows the overall structure of the subtasks of the Third Normal Form algorithm. As mentioned earlier, normalization codes encountered in the literature mainly make use of linked list structures with pointers and requires complex coding. In this work, the same algorithms are effectively implemented using only the Mathematica's basic list structure and the well-known set operations. These operations are Union[], Complement [], Intersection [], MemberQ[], Extract[], Append[], and Length[].

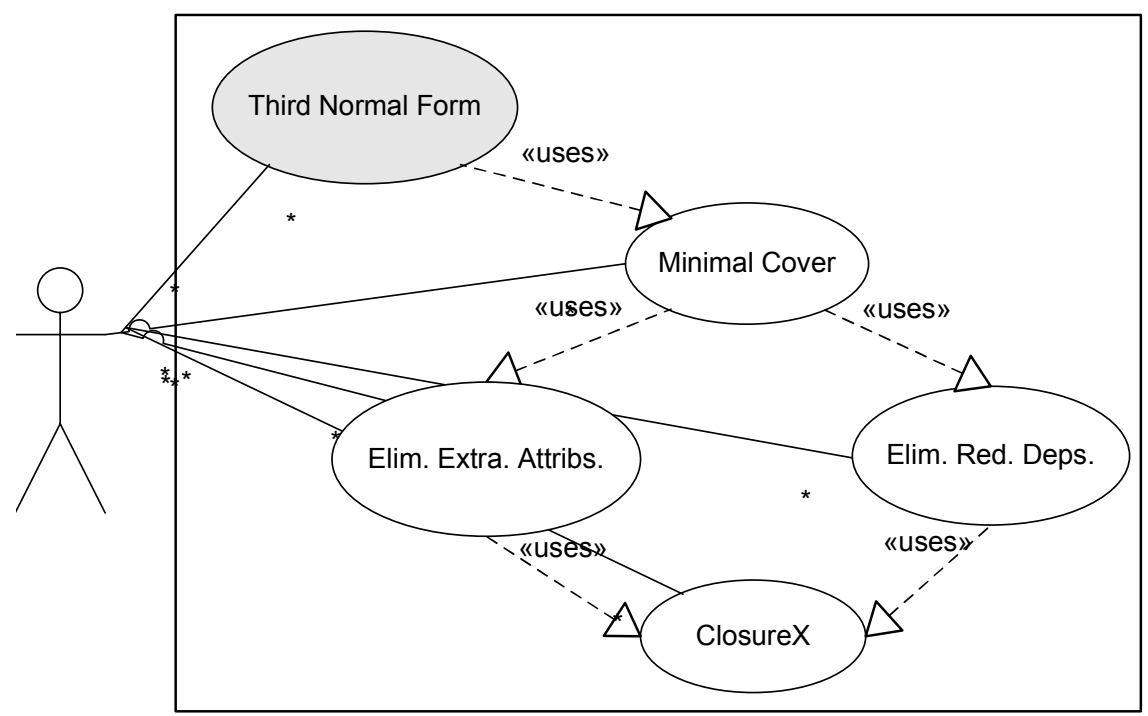

Fig. 1. Use Case Diagram for 3NF Modules

The tasks shown in Fig.1 are implemented effectively as Mathemamatica modules. As an illustration, the Mathematica code for the ThirdNormalForm only is given below. This program takes as input the minimal cover $[A G L, A G R]$ of the original set of FD's and generates a set of decompositions DL's each in 3NF.

ThirdNormalForm [AGL_, AGR_] : =

Module [ $\{i, k, m$, NewGL, NewGR , DR , DL , Index , TempGL , TempDL , TempGR , count $\}$,

$\mathrm{GL}=\mathrm{AGL} ; \mathrm{GR}=\mathrm{AGR} ; \mathrm{i}=1$; count $=1$;

While [count $<=$ Length $[$ AGL] \&\& Not $[G L==\{\}]$,

Index $=\{i\}$;

If $[$ Length $[\mathrm{GL}[[i]]]<=1, \mathrm{DL}=\{\mathrm{GL}[[i]]\}, \mathrm{DL}=\mathrm{GL}[[i]]]$;

TempDL=DL; $D R=\{G R[[i]]\} ;$ 


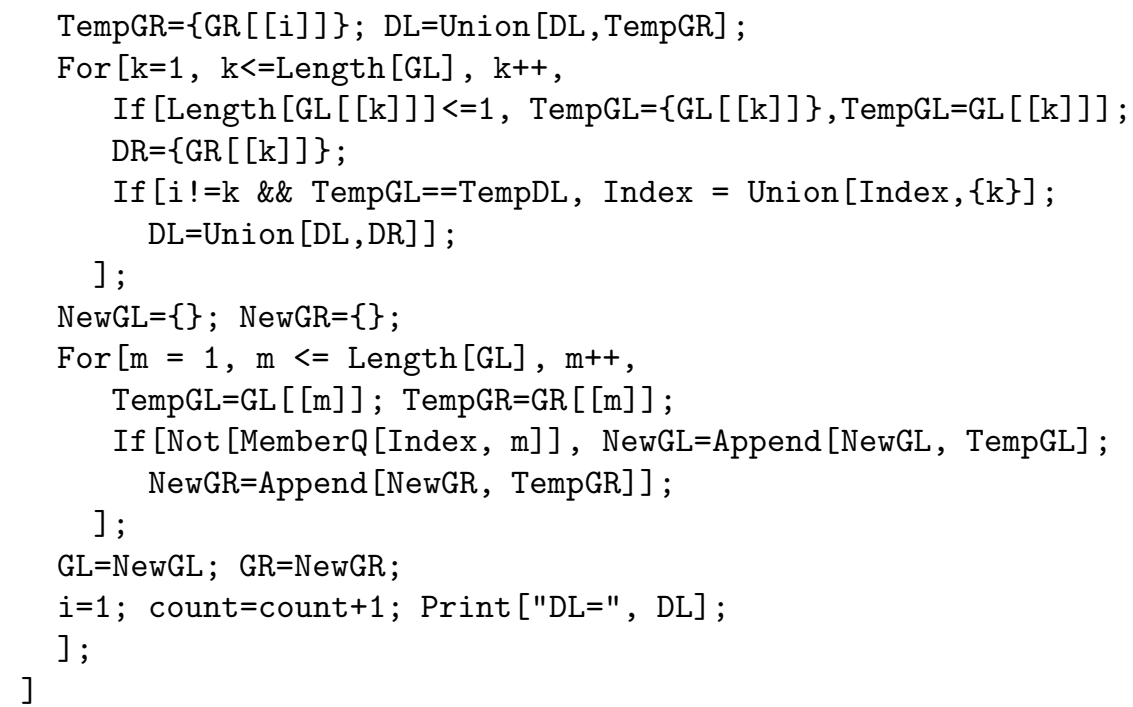

\subsection{Sample Runs}

A set of experiments are performed to check the correctness of the programs using benchmark tests found in the literature. In this section, the results of running the programs with two different benchmark schemas only are given.

Test 1. Consider the STUDENT DB schema and the FD's given by FD1, and FD2 above. Here, 3NF is violated for the STUDENT schema above, because of the transitive dependency between studentNo and officeNo. First of all, a minimal cover is to be obtained which requires the elimination of extraneous attributes followed by the elimination of redundant dependencies. In this case, there are no extraneous attributes. However, the redundant dependency studentNo $\rightarrow$ officeNo is automatically eliminated from FD2. The new set of FD's which is the minimal cover is obtained as follows:

$F D 1^{\prime}:$ studentNo $\rightarrow\{$ studentName, instructorId $\}$

$F D 2^{\prime}:$ instructorId $\rightarrow$ officeNo

By calling the ThirdNormalForm program with the minimal cover set above as input, the following $3 \mathrm{NF}$ decompositions are produced:

$S T U D E N T_{1}=\{$ studentNo, studentName, instructorId $\}$, $S T U D E N T_{2}=\{$ instructorId, officeNo $\}$

Test 2. This benchmark test is artificially produced in order to check the correctness of all the subtasks involved. The DB schema is $B M=\{a, b, c, d, e, g\}$ and a set of eleven FD's are given as follows:

$$
\begin{aligned}
& \{a, b\} \rightarrow c, c \rightarrow a,\{b, c\} \rightarrow d,\{a, c, d\} \rightarrow b, d \rightarrow e, d \rightarrow g,\{b, e\} \rightarrow c,\{c, g\} \rightarrow b, \\
& \{c, g\} \rightarrow d, \text { and }\{c, e\} \rightarrow g
\end{aligned}
$$



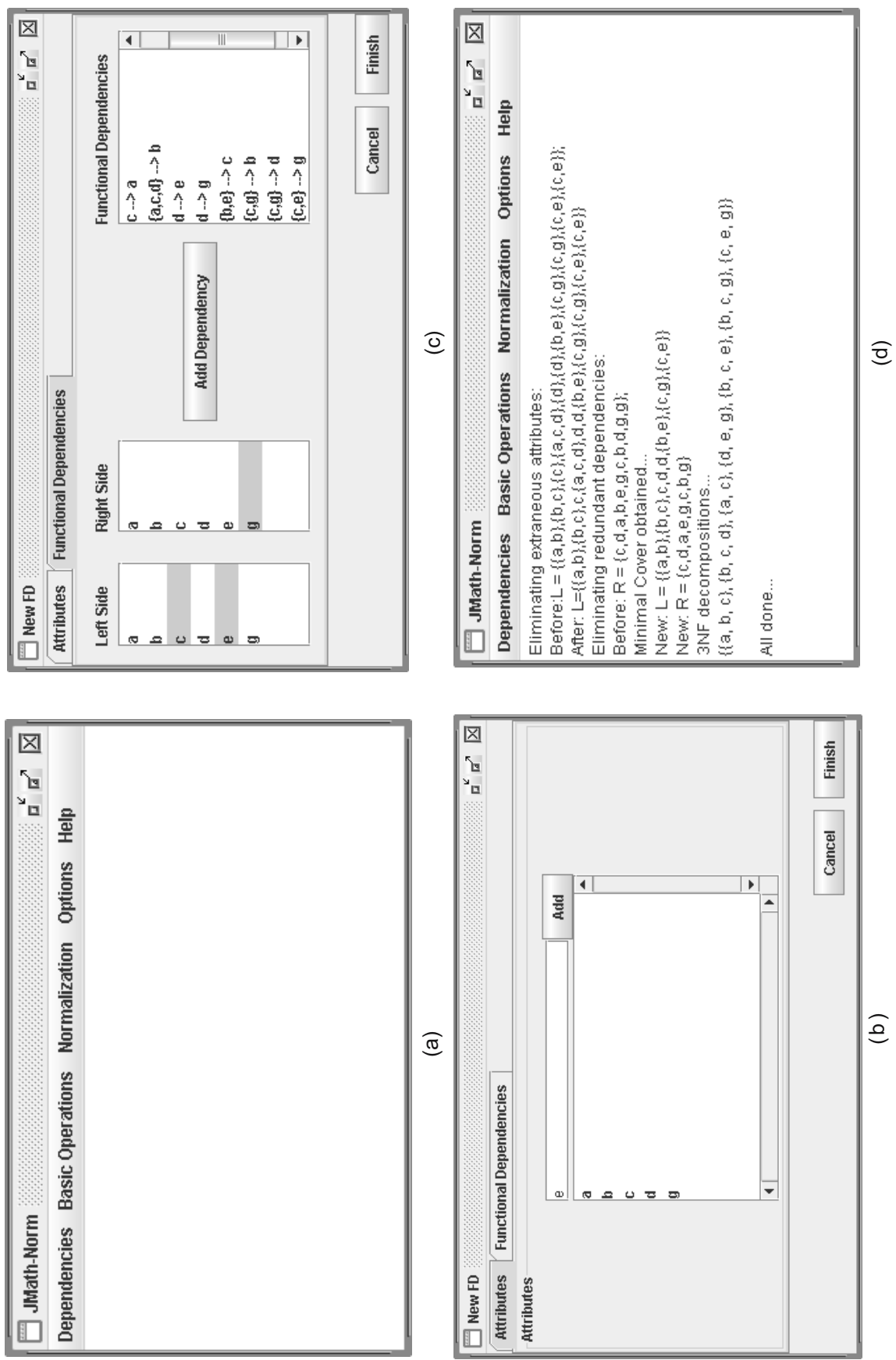

Fig. 2. JMath-Norm in Action (a) Main menu (b) Defining attributes (c) Specification of FD's (d) Normalization into 3NF 
The minimal cover module eliminates the extraneous attribute $a$ from $\{a, c, d\} \rightarrow b$, replacing it by $\{c, d\} \rightarrow b$. This FD set has 3 redundant dependencies, namely, $c \rightarrow a,\{c, d\} \rightarrow b$, and $\{c, g\} \rightarrow d$. Hence, all three is eliminated giving a minimal cover set with eight dependencies. $3 \mathrm{NF}$ decomposition sets as an output from ThirdNormalForm are obtained as follows:

$$
\{a, b, c\},\{b, c, d\},\{d, e, g\},\{b, c, e\},\{b, c, g\},\{a, c\} \text {, and }\{c, e, g\}
$$

\section{JMath-Norm: A Java Tool with Mathematica and JLink}

\subsection{System Architecture}

In this section, a prototype Java tool called JMath-Norm, developed by the authors is going to be illustrated. This tool is written in Java language with Java Link facility (JLink) of Mathematica which allows linking the Java code with the Mathematica kernel and execute the modules written in Mathematica. The main user interface is shown in Fig.2(a) which consists of submenus to manage FD's, basic normalization tasks, normalization to ThirdNormalForm, and others. Using JMath-Norm's Dependencies pull-down menu, the users may define a new set of FD's, open an existing one, save or edit FD's using a data entry dialog box. Basic normalization tasks such as Set Closure, and Minimal Cover can be carried out individually by utilizing the operations menu. 3NF form of a DB schema can be obtained from Normalization submenu.

\subsection{Using JMath-Norm}

The example given in Test. 2 above will be used to illustrate some of the features of JMath-Norm. The user starts by selecting New FD from the Dependencies pull-down menu to enter the attribute names one by one. This process is shown in Fig.2(b) below. The user then selects Functional Dependencies tab from the dialogue box, to efficiently define FD set by selecting attributes from the left and right pane respectively to enter the lhs and rhs of the FD set using the Add Dependency button(see Fig.2(c)).

After completing the specification of FD's, the user may either choose to select from a set of aforementioned basic normalization processes or directly obtain the $3 \mathrm{NF}$ decompositions by selecting $3 \mathrm{NF}$ from the Normalization tab of the main menu. The output for the later is shown in Fig.2(d).

\section{Conclusions}

In this paper, an efficient Mathematica implementation of basic DB normalization algorithms is presented. It was observed that, the implementation uses only Mathematica's collection operations. Previous implementations, however, are heavily based on linked lists and pointer structures which produces computationally inefficient systems. 
The Mathematica modules developed for the purpose are put together into a window based system JMath-Norm which provides interaction between the user and Mathematica. JMath-Norm is written in Java and utilizes JLink to derive the Mathematica kernel. By using the system user can arrange I/O into files which is displayed on user-friendly screens for which no Mathematica knowledge is actually required.

This prototype provides an interactive tool that is useful for teaching the normalization theory in a DB management course. The system, when completed, will provide modules for the other normalization forms and enable users to create DB tables automatically for a specific DBMS chosen.

\section{References}

1. Elmasri, R. and Navathe, S.B.: Fundamentals of Database Systems, 4th Ed., Addison Wesley (2003).

2. Beeri, C. and Bernstein, P.A.: Computational Problems Related to the Design of Normal Form Relational Schemas, ACM Transactions on Database Systems, Vol.4, No.1 (1979) 30-45.

3. Ceri, S. and Gottlob, G.: Normalization of Relations and Prolog, Communications of the ACM, Vol.29, No.6 (1986)

4. Welzer, W., Rozman, I. and Gyrks, J.G.: Automated Normalization Tool, Microprocessing and Microprogramming, Vol.25, (1989) 375-380.

5. Diederich, J. and Milton, J.: New Methods and Fast Algorithms for Database Normalization, ACM Transactions on Database Systems, Vol.13, No.3(1988) 339365 .

6. Diederich, J.: Minimal Covers Revisited: Correct and Efficient Algorithms, SIGMOD Record, Vol.20, No.1 (1991)

7. Hallasy, B.: Normal Forms and Normalization: Practical Designer's View, Information and Software Technology, Vol.25, No.6 (1991) 451-461.

8. Lucchesi, C.L. and Osborn, S.L.: Candidate Keys for Relations, Journal of Computer and System Sciences, Vol.17, No.2 (1978) 270-279.

9. Westlend, J.C.: Economic Incentives for Database Normalization, Information Processing and Management, Vol.28, No.5 (1992)647-662.

10. Lee, H.: Justifying Database Normalization: A Cost/Benefit Model, Information Processing and Management, Vol.31, No.1 (1995) 56-67.

11. Millet, I.: Normalization on the Fly, Journal of Systems Software, Vol.40 (1998) $85-88$.

12. Vincent, M.W. and Srivinasan, B.: A Note on Relation Schemas Which Are in 3NF But Not in BCNF, Information Processing Letters, Vol.48 (1993) 281-283.

13. Du, H. and Wery, L.: Micro: A Normalization Tool for Relational Database Designers, Journal of Network and Computer Applications, Vol.22, (1999) 215-232.

14. Wolfram, S.: The Mathematica Book, 4th Ed., Wolfram Media/Cambridge University Press (1999).

15. Bernstein, P.A.: Synthesizing Third Norm Relations from Functional Dependencies, ACM Trans. Database Systems, Vol.1, No.4 (1976) 277-298. 